\title{
Functional hierarchy for tactile processing in the visual cortex of sighted adults
}

\author{
Łukasz Bola $^{\text {a,b,2,3,*, Jacek Matuszewski }}{ }^{\text {a,2 }}$, Michał Szczepanik ${ }^{\text {a }}$, Dawid Droździel ${ }^{\text {a }}$,

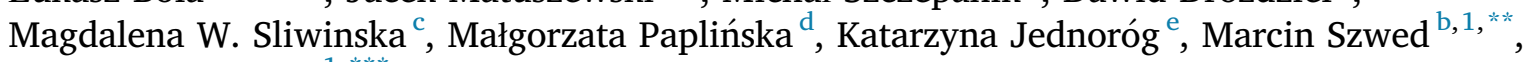 \\ Artur Marchewka ${ }^{a, 1, * * *}$

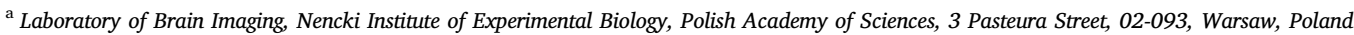 \\ ${ }^{\mathrm{b}}$ Institute of Psychology, Jagiellonian University, 6 Ingardena Street, 30-060, Krakow, Poland \\ ${ }^{\mathrm{c}}$ Department of Psychology, University of York, Heslington, YO10 5DD, York, UK \\ ${ }^{\mathrm{d}}$ The Maria Grzegorzewska University, 40 Szczęśliwicka Street, 02-353, Warsaw, Poland \\ ${ }^{\mathrm{e}}$ Laboratory of Language Neurobiology, Nencki Institute of Experimental Biology, Polish Academy of Sciences, 3 Pasteura Street, 02-093, Warsaw, Poland
}

\section{A R T I C L E I N F O}

\section{Keywords:}

Perception

Cross-modal interactions

Braille

Visual cortex

Somatosensory cortex

TMS

\begin{abstract}
A B S T R A C T
Perception via different sensory modalities was traditionally believed to be supported by largely separate brain systems. However, a growing number of studies demonstrate that the visual cortices of typical, sighted adults are involved in tactile and auditory perceptual processing. Here, we investigated the spatiotemporal dynamics of the visual cortex's involvement in a complex tactile task: Braille letter recognition. Sighted subjects underwent Braille training and then participated in a transcranial magnetic stimulation (TMS) study in which they tactually identified single Braille letters. During this task, TMS was applied to their left early visual cortex, visual word form area (VWFA), and left early somatosensory cortex at five time windows from 20 to $520 \mathrm{~ms}$ following the Braille letter presentation's onset. The subjects' response accuracy decreased when TMS was applied to the early visual cortex at the 120-220 ms time window and when TMS was applied to the VWFA at the 320-420 ms time window. Stimulation of the early somatosensory cortex did not have a time-specific effect on the accuracy of the subjects' Braille letter recognition, but rather caused a general slowdown during this task. Our results indicate that the involvement of sighted people's visual cortices in tactile perception respects the canonical visual hierarchy-the early tactile processing stages involve the early visual cortex, whereas more advanced tactile computations involve high-level visual areas. Our findings are compatible with the metamodal account of brain organization and suggest that the whole visual cortex may potentially support spatial perception in a task-specific, sensoryindependent manner.
\end{abstract}

\section{Introduction}

Until recently, perception via different sensory modalities was thought to be supported by largely separate brain systems - it was generally assumed that the visual cortex processes solely visual input, the somatosensory cortex processes solely tactile input, and so on (e.g., Fig. 18-2 in Kandel et al., 2012). Departures from this rule and the "unmasking" of cross-modal interactions during perceptual processing were reported mainly following sensory loss or injury (Bavelier and Neville, 2002; Lomber et al., 2011; Merabet and Pascual-Leone, 2010; Rauschecker, 1995; Sur et al., 1990). However, a growing number of studies demonstrate that tactile and auditory tasks involve the visual cortex even in typical adults (Amedi et al., 2007, 2001; Campus et al., 2017; Deshpande et al., 2010; Eck et al., 2016, 2013; Hagen et al., 2002;

\footnotetext{
* Corresponding author. Institute of Psychology, Jagiellonian University, 6 Ingardena Street, 30-060, Krakow, Poland.

$* *$ Corresponding author.

$* * *$ Corresponding author.

E-mail addresses: bolalukasz@gmail.com (Ł. Bola), mfszwed@gmail.com (M. Szwed), a.marchewka@nencki.gov.pl (A. Marchewka).

1 These authors share senior authorship.

2 Equally contributing authors.

${ }^{3}$ Present address: Department of Psychology, Harvard University, 33 Kirkland Street, Cambridge, MA, 02138, USA.
} 
Kim and Zatorre, 2011; Lacey et al., 2014, 2010; Merabet et al., 2008, 2006; Poirier et al., 2005; Saito et al., 2006; Sathian et al., 2011, 1997; Siuda-Krzywicka et al., 2016; Stilla and Sathian, 2008; Tal et al., 2016; Zangenehpour and Zatorre, 2010) and that this involvement is functionally relevant (Amemiya et al., 2017; Merabet et al., 2008, 2004; Siuda-Krzywicka et al., 2016; Zangaladze et al., 1999). These findings suggest that cross-modal interactions between sensory systems are not an exception, possible only in the context of sensory deprivation or brain injury, but are rather a general mechanism that supports human perception.

The exact way in which the functioning visual cortex interacts with other sensory systems during tactile and auditory perception remains to be elucidated. Nevertheless, previous studies have already indicated that these interactions might follow a specific spatial pattern. In sighted adults, early visual areas are recruited through relatively simple tactile and auditory discrimination, such as through comparing the shape of single Braille characters, exploring various textures, or perceiving noise bursts (Eck et al., 2013; Merabet et al., 2008; Sathian et al., 2011; Stilla and Sathian, 2008; Zangenehpour and Zatorre, 2010; see also Merabet et al., 2006; Saito et al., 2006). Tasks that require a more complex perceptual analysis, such as tactile or auditory object recognition, tactile or auditory motion perception, or whole-word Braille reading, activate relevant high-level visual regions in the ventral and dorsal visual streams (Amedi et al., 2007, 2001; Hagen et al., 2002; Kim and Zatorre, 2011; Lacey et al., 2014, 2010; Poirier et al., 2005; Siuda-Krzywicka et al., 2016). These results suggest that, in a sighted person, the visual cortex's involvement in tactile and auditory tasks might respect the typical visual processing hierarchy (Reddy and Kanwisher, 2006; Riesenhuber and Poggio, 2000; Rolls, 2001); this possibility is also suggested by our recent studies (Bola et al., 2017a; Siuda-Krzywicka et al., 2016), which demonstrated that, in sighted adults, learning to read Braille-a tactile task encompassing both spatial and linguistic processing — results in both the anatomical reorganization of the early visual cortex as well as the functional recruitment of the left ventral visual cortex, especially the visual word form area (VWFA; Dehaene and Cohen, 2011; Price and Devlin, 2011). Based on the conjecture described above, one may expect that these visual regions are involved in tactile Braille reading in a hierarchical manner to support the different types of computations necessary to accomplish this task. The early visual cortex would be involved first to perhaps support the construction of a spatial representation of Braille dots and signs; only then would the Braille reading involve the high-level ventral visual cortex, which may support the creation of an abstract representation of a Braille letter (see, e.g., the visual reading model proposed by Dehaene et al., 2005). Here, we put these predictions to the test.

A group of seventeen sighted subjects, independent of the group described in our previous studies (Bola et al., 2017b, 2017a; 2016; Siuda-Krzywicka et al., 2016), received training in tactile Braille reading for eight months and then enrolled in a chronometric transcranial magnetic stimulation (TMS) experiment. In chronometric TMS, magnetic pulses are applied at specific time windows to investigate at which time each brain area is involved in a given task (reviewed in Pascual-Leone et al., 2000). In this study, we used this method to test whether and at which point neural activity in the early visual cortex and the ventral visual cortex is causally linked with successful tactile Braille letter recognition. Subjects were asked to read aloud single Braille letters presented in the tactile modality while TMS was applied to the left early visual cortex, the VWFA, or the left early somatosensory cortex at five different time windows spanning from 20 to $520 \mathrm{~ms}$ following the Braille letter presentation's onset (Fig. 1).

Previous studies suggest that Braille letter recognition should not be disrupted by the stimulation of visual areas applied as early as $20-120 \mathrm{~ms}$ following the letter presentation's onset (i.e., the earliest time window in our experiment). Zangaladze et al. (1999), for example, reported that the visual cortex's most pronounced involvement in tactile perception occurs well after this time (i.e., $150-200 \mathrm{~ms}$ following the stimulus presentation) even in the case of a relatively low-level tactile task (discriminating grating orientations). We thus hypothesized that, relative to the earliest TMS time window, Braille letter recognition will be specifically disrupted by: (1) TMS applied to the early visual cortex in intermediate (i.e., $120-220$ or $220-320 \mathrm{~ms}$ ) but not in late (i.e., $320-420$ or $420-520 \mathrm{~ms}$ ) time windows; and (2) TMS applied to the VWFA in late (320-420 or
A
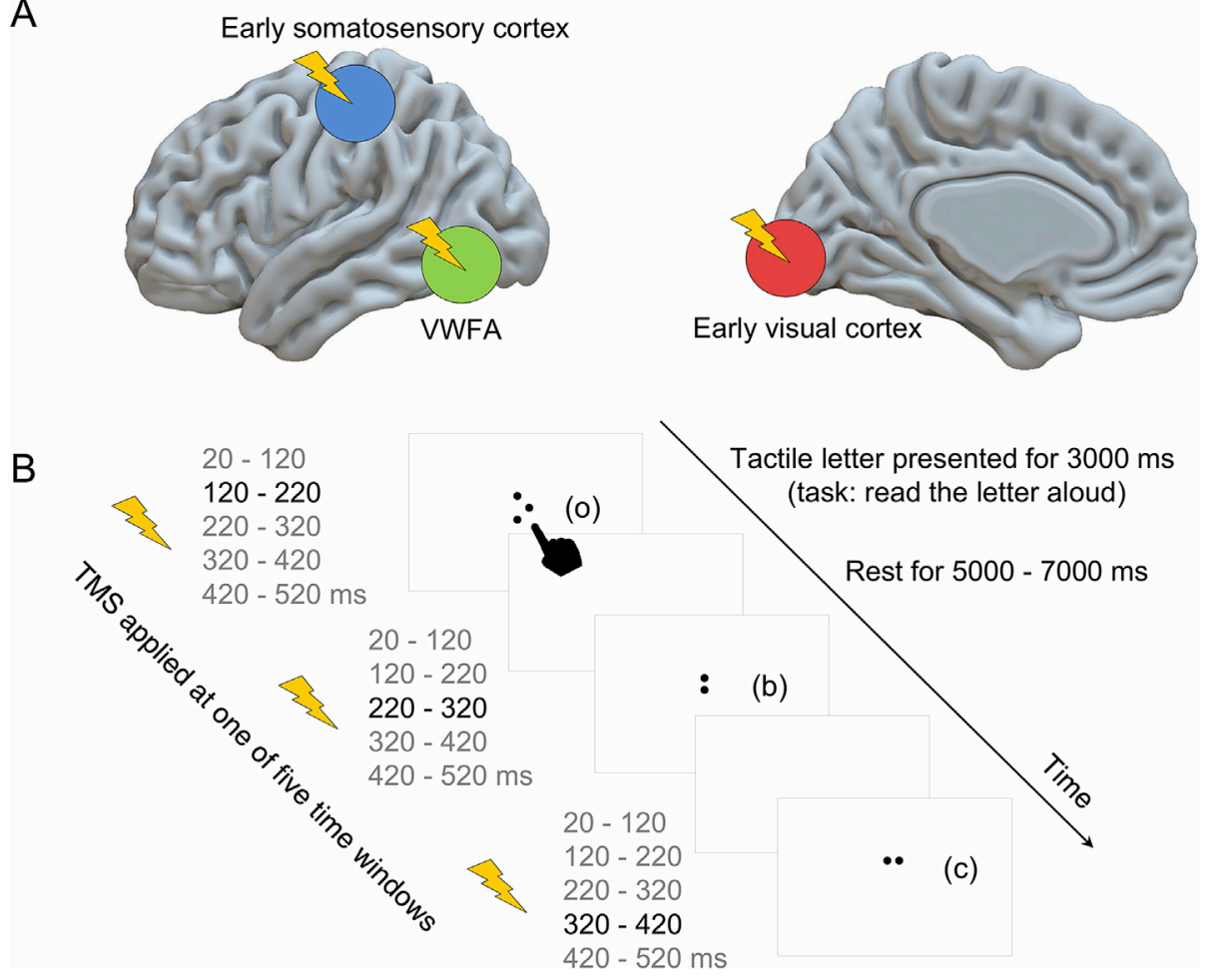

Fig. 1. Experimental design. (A) Subjects read aloud single Braille letters presented in the tactile modality while TMS was applied to their left early visual cortices, visual word form areas (VWFAs,) and left early somatosensory cortices; (B) each trial consisted of a 3-s Braille letter presentation, followed by a 5- to 7-s rest period with no stimuli presented. During each trial, three TMS pulses with an interpulse interval of $50 \mathrm{~ms}(20 \mathrm{~Hz})$ were applied at one of five time windows - 20-120, $120-220,220-320,320-420$, or $420-520 \mathrm{~ms}-$ following the Braille letter presentation's onset. Note that the representation of TMS sites in the figure is schematic and reflects neither their exact localization nor the exact focus of TMS in the experiment. 
$420-520 \mathrm{~ms})$ but not intermediate $(120-220$ or $220-320 \mathrm{~ms})$ time windows. Establishing this temporal double dissociation will constitute evidence of the visual cortex's hierarchical involvement in tactile processing in a way that respects the canonical visual processing hierarchy from early to high-level visual cortices.

\section{Materials and methods}

\subsection{Subjects}

Twenty-one healthy, right-handed female subjects were initially recruited for the study (mean age $=23.7$ years; $\mathrm{SD}=3.3$ years; range $=20-31$ years). To ensure appropriate statistical power, we decided to recruit at least fifteen to twenty subjects prior to the data collection-a sample size that has been proven sufficient for detecting TMS effects on reading and language processing (Pattamadilok et al., 2015; Schuhmann et al., 2012; Sliwinska et al., 2015). All subjects were native Polish speakers and had normal or corrected-to-normal vision. They were students studying special education and specializing in blindness and related disabilities who were visually familiarized with Braille signs as part of their curriculum. As in our previous studies (see, e.g., Bola et al., 2016; Siuda-Krzywicka et al., 2016), subjects were recruited from such a population for two reasons: (1) they were highly professionally motivated to participate in the tactile Braille reading course and (2) their familiarity with visual Braille reading was expected to facilitate a process of learning that would result in their ability to recognize Braille letters by touch. Interestingly, behavioral tests performed upon the tactile Braille training's onset revealed that the subjects' visual familiarity with Braille had only a limited influence on their initial ability to tactually read this script. While most subjects were able to recognize some Braille letters in the tactile modality, only five managed to read even a single word in $1 \mathrm{~min}$ (see Supplementary Information for the comparison of subjects' visual and tactile Braille reading abilities). Apart from the TMS experiment reported in this paper, the subjects participated in a longitudinal magnetic resonance imaging (MRI) study, the results of which will be described in a separate publication. All tests and procedures described in this paper were approved by the Committee for Research Ethics of the Institute of Psychology of the Jagiellonian University (approval granted on 02/22/2016). Informed consent and consent to publish were obtained from each subject prior to their testing.

During the TMS experiment, four subjects were excluded because they found the VWFA stimulation uncomfortable, even when its intensity was reduced from $110 \%$ (target intensity in the study; see Section 2.5 ) to $100 \%$ of their resting motor threshold; $100 \%$ is the lowest intensity that has proven to be effective in interfering with neural processing in this area (Duncan et al., 2010; Pattamadilok et al., 2015, 2010; Siuda-Krzywicka et al., 2016). Thus, data from the remaining seventeen subjects (mean age $=24$ years; $\mathrm{SD}=3.4$ years; range $=20-31$ years) were included in the analysis.

The reason why female subjects were exclusively recruited was related to the gender distribution in the special education student population in Poland, wherein such studies are primarily undertaken by women. It is important to note that previous work has demonstrated the existence of gender differences in the Braille reading abilities of visually impaired subjects (Argyropoulos and Papadimitriou, 2015). Thus, it is possible that the inclusion of only female subjects modulated either the Braille training's behavioral outcomes or the overall performance in the TMS experiment. Nevertheless, such modulation would be orthogonal to effects of interest in our study. It is unlikely that gender influences mechanisms of cross-modal interactions in the brain or affects how TMS impacts these mechanisms.

\subsection{Tactile Braille reading course and behavioral tests}

Before participating in the TMS experiment, all subjects completed an eight-month-long tactile Braille reading course and were administered tactile Braille word and tactile Braille letter reading tests both prior to and following the course in a manner similar to that of our previous studies (for a detailed description, see Bola et al., 2016; Siuda-Krzywicka et al., 2016). In addition, their visual Braille reading skills were tested both prior to and following the tactile Braille reading course. These tests involved reading aloud in the visual modality as many Braille words as possible within $1 \mathrm{~min}$. A list of 116 unrelated Polish words was employed, and both the word list and a testing procedure were adapted from a standard Polish reading speed test designed for school-aged children (seven to twelve years; Konopnicki, 1961).

\subsection{TMS study: task and stimuli}

In the TMS experiment, subjects were instructed to read aloud single Braille letters presented in the tactile modality using an Active Star display (HandyTech, Horb-Nordstetten, Germany). Single letter recognition was chosen rather than whole-word reading to minimize withinsubject and between-subject variance in performance, which may mask TMS effects-especially in chronometric designs. At the same time, reading aloud ensured that subjects were truly accessing a letter's representation and were not solving the task based solely on a low-level spatial representation of dots.

Each trial consisted of a 3-s-long Braille letter presentation, followed by a 5- to 7-s rest period with no stimuli presented on the display. Subjects were asked to identify Braille letters with the right-hand index finger. Each subject's finger was placed upon the Braille display before the letter was presented, and subjects were free to tactually explore a letter after it was presented. Subjects were not blindfolded, and no instructions were given regarding whether they should keep their eyes closed or open. This choice was motivated by two reasons: (1) our pilot experiments indicated that blindfolding subjects decreases the precision of TMS and, consequently, the quality of collected data (a TMS coil easily slips from a blindfold, especially during the early visual cortex stimulation; subjects' facial movements move a blindfold, which in turn moves neuronavigation trackers attached to subjects' heads; wearing a blindfold during a relatively long experiment increases subjects' discomfort); and (2) this design allowed us to test whether or not the tactile recognition of Braille letters can involve the visual cortex when the visual input is unconstrained - that is, in a situation that arguably resembles sighted people's everyday perceptual functioning more closely than do experiments that require blindfolding. The Braille display was covered to prevent subjects from visually recognizing stimuli. Vocal responses were recorded via a microphone for $4 \mathrm{~s}$ following the Braille letter presentation's onset. Overall, 300 trials were administered to each subject. Trials were divided into three equal runs, corresponding to three TMS sites. Within each run, trials were further divided into five subsets, corresponding to five TMS time windows. Consequently, all experiment trials were divided into fifteen equal subsets ( 3 TMS sites x 5 TMS time windows), each of which included twenty trials.

During Braille training, the subjects' tactile recognition of Braille letters was trained in a specific order. The first half of the Polish alphabet (sixteen letters, which are generally easier to recognize in the Braille alphabet: A, B, C, D, E, I, K, L, Ł, M, O, P, S, T, U, Y) was introduced at the beginning of the course, while the second half of the alphabet was introduced after the subjects' recognition of the first half was mastered (see Bola et al., 2016). To increase within-subject and between-subject consistency in performance, only letters that were introduced at the beginning of the Braille reading course-those that were practiced the most extensively - were employed in the TMS experiment as stimuli. The letter "A" was excluded from the list of stimuli because it is the only Braille letter that consists of one dot and may be processed using different mechanisms than other Braille letters (e.g., the recognition of "A" does not involve combining dots into a coherent, spatial representation as the recognition of other Braille letters does); as a result, fifteen letters were used as stimuli (B, C, D, E, I, K, L, Ł, M, O, P, S, T, U, Y). Within each condition, the same set of Braille letters was presented. The letters B, C, E, 
$\mathrm{K}, \mathrm{L}, \mathrm{M}, \mathrm{O}, \mathrm{S}, \mathrm{T}$, and $\mathrm{Y}$ were presented once per condition, while the letters $\mathrm{D}, \mathrm{I}, \mathrm{E}, \mathrm{P}$, and $\mathrm{U}$ were randomly chosen to be presented twice in order to reach a target number of trials per condition (i.e., twenty trials; see above). The letter presentation's order was randomized for each subject. The randomization procedure was constrained by the rule that the same letter could not be presented twice in a row. Stimulus presentation and response recording were controlled using a program written in Python that relied upon the PsychoPy package (Peirce, 2007).

\subsection{Localization of TMS sites}

During the TMS experiment, the left early visual cortex, the VWFA, and the left early somatosensory cortex were targeted using a neuronavigation system. Prior to the experiment, those sites were localized and marked on each subject's MRI scan. The early visual cortex and the early somatosensory cortex were localized based on each subject's brain anatomy. The early visual cortex was defined as a posterior termination of the calcarine sulcus (Chambers et al., 2013; Merabet et al., 2008). The early somatosensory cortex was marked within the postcentral gyrus, roughly $1-2 \mathrm{~cm}$ posteriorly from "the omega knob" in the precentral gyrus (i.e., a canonical location of the hand area in the primary motor cortex; Merabet et al., 2004; Vidoni et al., 2010). In contrast, the VWFA was localized using individual, functional MRI activations during tactile and visual lexical decision tasks acquired prior to the TMS study. The localization tasks were part of a separate longitudinal MRI study performed on the same group of subjects, which will be described in another publication (see Supplementary Information for details that might be relevant to the present study). In order to localize the VWFA in the TMS experiment, a two-step procedure was employed. Firstly, brain activations enhanced by the visual lexical decision task (performed in the Latin alphabet) - a task that is known to strongly activate the VWFA (e.g., Rauschecker et al., 2011)—relative to the detection of hash signs in the string of consonants were employed to broadly localize an area sensitive to orthographic processing in the left ventral occipitotemporal region. Secondly, an activation peak enhanced by the tactile lexical decision task (performed in the Braille alphabet) relative to the detection of meaningless Braille signs in the string of consonants written in the Braille alphabet was used to define the stimulation's localization within the region of interest obtained during the first step. To verify the accuracy of our VWFA localization procedure, single-subject data were normalized to the Montreal Neurological Institute (MNI) space, and the chosen VWFA coordinates were averaged across subjects. The mean MNI coordinates obtained using this procedure (mean \pm standard error of the mean: $\mathrm{x}=-39 \pm 1, \mathrm{y}=-62 \pm 1, \mathrm{z}=-11 \pm 1$ ) agreed with the VWFA's location as reported in the literature (e.g., Cohen et al., 2002; Glezer et al., 2009), which indicates that our localization procedure was accurate.

TMS was expected to affect the subjects' Braille letter recognition at different time points across the three sites (see Section 2.9); consequently, the sites could serve one another as inherent control, and the inclusion of a separate control site was not required.

\subsection{TMS protocol}

TMS was administered using a MagPro X100 stimulator (MagVenture, Hückelhoven, Germany) with a 70-mm figure-eight coil. Stimulation was guided with a Brainsight 2 neuronavigation system (Rogue Research, Montreal, Canada) and a Polaris Vicra infrared camera (Northern Digital, Waterloo, Canada). In each trial, three pulses with an interpulse interval of $50 \mathrm{~ms}(20 \mathrm{~Hz})$ were applied at one of five time windows, namely 20-70-120 ms, $120-170-220 \mathrm{~ms}, 220-270-320 \mathrm{~ms}, 320-370-420 \mathrm{~ms}$, or $420-470-520 \mathrm{~ms}$, following the Braille letter presentation's onset. Stimulation was administered in relatively wide time windows lasting $100 \mathrm{~ms}$ rather than the $10-40 \mathrm{~ms}$, which is the usual TMS time window width in studies of visual reading (Amassian et al., 1989; Duncan et al., 2010; Salminen-Vaparanta et al., 2012). This was done in order to account for the difficult nature of tactile reading and expected between-subject variability in the task performance. Previous studies have shown that chronometric TMS with the adjusted time window width can be successfully applied to interfere with complex mental processes, even when between-subject variability is high (e.g., Sack et al., 2005).

TMS intensity was initially set to $110 \%$ of each participant's resting motor threshold. The motor threshold was indicated by the lowest stimulator output needed to elicit a visible twitch of the relaxed hand in at least five of ten trials during the contralateral primary motor cortex stimulation. The average individual motor threshold was $37 \%(\mathrm{SD}=6 \%$; range $24-48 \%$ ) of the maximum stimulator output power. Prior to the actual data collection, TMS was applied with a target intensity to each site, and subjects were surveyed for any side effects of the stimulation. Six subjects reported hand movements during the early somatosensory cortex stimulation, and five subjects reported uncomfortable head muscle twitches during the VWFA stimulation. In these cases, the stimulation's intensity for a given site was reduced to $100 \%$ of the individual motor threshold. As a result, in the actual TMS experiment, hand movements during the early somatosensory cortex stimulation were neither reported by any participant nor observed by an experimenter. For the VWFA stimulation, residual head muscle twitches were at times observed, even when the TMS intensity was adjusted. Provided that this condition was comfortable for a given subject, the study was performed normally. Since the VWFA stimulation was expected to interfere with tactile Braille letter recognition at a specific time window, TMS within this site at other time windows controlled for these peripheral effects. Subjects did not report any side effects during the early visual cortex stimulation.

The TMS time windows' order was randomized for each subject and experimental run. The randomization procedure was constrained by the rule that, in adjacent experimental trials, TMS was applied at adjacent time windows (e.g., $120-170-220 \mathrm{~ms}, 20-70-120 \mathrm{~ms}, 120-170-220 \mathrm{~ms}$, $220-270-320 \mathrm{~ms}, 320-370-420 \mathrm{~ms})$. This assured that timing differences between time windows-especially the early and late time windows-were difficult to notice (see also Pattamadilok et al., 2015; Sliwinska et al., 2012). The TMS sites' order was counterbalanced across subjects.

\subsection{MRI protocol}

MRI data were acquired using a Siemens Trio 3T scanner and a twelve-channel coil. A high-resolution, structural T1-weighted image was acquired with the following parameters: field of view: $256 \times 256 \mathrm{~mm}$, isometric voxel size: $1 \mathrm{~mm}$, TR: 2530 , TE: 3.32, flip angle: $7^{\circ}, 176$ slices. Functional data were acquired using an echo planar imaging pulse sequence with the following parameters: field of view: $216 \times 216 \mathrm{~mm}$, isometric voxel size: $3 \mathrm{~mm}$, matrix $72 \times 72$, TR: $2500 \mathrm{~ms}$, TE: $28 \mathrm{~ms}$, flip angle: $80^{\circ}$, 41 slices in the AC-PC plane with an odd interleaved order.

\subsection{Procedure}

After providing informed consent and completing a safety screening questionnaire, the subjects were familiarized with TMS and the neuronavigation system. The structural MRI scan with the marked TMS target sites was subsequently co-registered to a participant's head. Next, the resting motor threshold was measured with single TMS pulses administered to the hand area in the left primary motor cortex. Afterwards, two short training sessions were performed without and with TMS, respectively, to familiarize subjects with the task and the triple-pulse TMS protocol. The actual TMS experiment was subsequently conducted. All three target sites were tested one by one in three separate runs and with 5-min breaks between each run. Prior to each run, TMS was applied to the target site to test for potential side effects of the stimulation (see Section 2.5). The whole procedure lasted approximately $120 \mathrm{~min}$. 


\subsection{Data preprocessing}

For every trial, accuracy and reaction times were manually marked from the recorded vocal responses in a blind fashion (i.e., a judge did not know to which experimental condition a given trial belonged). One trial was excluded from all further analyses due to an unexpected interruption of the experimental procedure that took place during this trial; thus, 5099 trials were included in the further steps. Unusually accelerated or delayed responses, defined as those that were $2.5 \mathrm{SD}$ faster or slower than individual subjects' means within each experimental condition, were treated as missing responses to minimize variance in the data and improve statistical power (seventy trials; $1.4 \%$ of the data). Additionally, a log transformation was applied to individual reaction time data to ensure the distributions' normality (McDonald, 2009).

The Audacity software (www.audacityteam.org) and in-house Python scripts were employed to perform accuracy and reaction time marking. All statistical analyses were performed in the SPSS 25 package (IBM, USA).

\subsection{Data analysis: accuracy}

Given that the analysis of variance (ANOVA) applied to variables with binomial distribution (e.g., subjects' accuracy quantified binomially as either a correct or incorrect response) might produce spurious results (Jaeger, 2008), a statistical analysis of the accuracy data was performed within the generalized linear mixed model (GLMM). All valid trials $(\mathrm{N}=5099$; see Section 2.8) were entered into the GLMM and were modelled as a binomial dependent variable using a logit link function (correct answers vs. all errors-i.e., incorrect and missing answers modelled jointly; see Supplementary Information for additional analyses, in which incorrect and missing answers were modelled separately). The TMS site (the early visual cortex, VWFA, and early somatosensory cortex), the TMS time window (0-70-120, 120-170-220, 220-270-320, $320-370-420$, and $420-470-520 \mathrm{~ms}$ ), the TMS site $\mathrm{x}$ TMS time window interaction, and an intercept were included in the model as fixed effects. Additionally, a subject intercept was included as a random effect with the "variance component" covariance type, to account for interpersonal variability. The early somatosensory cortex, the first TMS time window, and their combination were used as reference categories for TMS site, TMS time window, and TMS site $\mathrm{x}$ TMS time window interaction coefficients, respectively. The model was estimated using the SPSS "robust estimation" procedure to account for potential violations of the model assumptions and with degrees of freedom fixed for all tests.

Pairwise comparisons were performed on estimated marginal means reflecting the probability of a subject's correct recognition of a Braille letter under a given condition. A significant TMS time window $\mathrm{x}$ TMS site interaction effect was investigated across both TMS time windows and TMS sites. In comparisons between TMS time windows within each site, the first time window $(20-120 \mathrm{~ms}$ following the Braille letter presentation's onset) was compared to every other time window. This choice was motivated by several reasons. Firstly, such an early stimulation of the visual cortex is unlikely to affect tactile processes in sighted subjects (Zangaladze et al., 1999; see Section 1). We thus expected that TMS applied to the early visual cortex and the VWFA in later time windows would disrupt subjects' performances relative to the stimulation in the first time window. Secondly, the early somatosensory cortex is critical for the initial tactile perception stage. It was demonstrated, for example, that TMS applied to this site $30 \mathrm{~ms}$ following the presentation of a tactile grating strongly interfered with judgments on its orientation (Zangaladze et al., 1999). Based on the early somatosensory cortex's location in the tactile processing hierarchy, one may expect that a disruptive effect of TMS applied to this site should be observed in the first time window and vanish in later time windows; our planned comparisons allowed us to test this prediction (i.e., test for an increase in accuracy in later time windows relative to the accuracy in the first time window). Thirdly, contrasts made against a TMS time window in which no effect is expected provide greater control for unspecific stimulation effects than contrasts against no-TMS or sham conditions because they control for both noise and tactile sensations (see De Graaf and Sack, 2011; Duncan et al., 2010; Pattamadilok et al., 2015; Sliwinska et al., 2012). Within each TMS site, a Bonferroni correction was applied to correct the results for four comparisons that were made (i.e., the first time window vs. every other time window). Direct comparisons between time windows other than the first were not performed because we did not have any specific hypothesis regarding such contrasts (for a similar analytical strategy, see, e.g., Duncan et al., 2010; Pattamadilok et al., 2015; Sliwinska et al., 2012). In comparisons between TMS sites within each time window, all sites were compared with one another. Consequently, a Bonferroni correction was applied to correct the results for three comparisons that were made within each time window.

An additional analysis was performed to specifically test our hypothesis regarding the temporal double dissociation between effects of the early visual cortex stimulation and the VWFA stimulation on the subjects' Braille letter recognition accuracy. Given that this hypothesis did not concern the early somatosensory cortex, this TMS site was excluded, thus resulting in a 2 TMS site x 5 TMS time window GLMM model (3399 trials included; all other model parameters were maintained as they were in the main analysis). In order to provide a stringent test for the double dissociation, the pairwise comparisons' results were corrected for all tests performed, considered jointly across factors (i.e., the results were corrected for thirteen comparisons: four comparisons between the first and every other TMS time window within each TMS site and five comparisons between TMS sites). A correction for multiple comparisons was performed using the false discovery rate (FDR; Benjamini and Hochberg, 1995), which provides more balanced p-value estimates than does a Bonferroni correction when the number of comparisons is high.

\subsection{Data analysis: reaction time}

Median reaction times from correct responses were entered into a repeated-measure $3 \times 5$ ANOVA with the TMS site (the early visual cortex, VWFA, and early somatosensory cortex) and the TMS time window (0-70-120, 120-170-220, 220-270-320, 320-370-420, and $420-470-520 \mathrm{~ms}$ ) as within-subject factors. Pairwise comparisons were performed using the same analytical logic as was described above. While the actual reaction time analysis was performed on log-transformed data, reaction times prior to log transformation are reported in the text and presented in Fig. 4 in order to enhance the presented results' interpretability.

\subsection{Data availability}

The accuracy and reaction time data, obtained as a result of the marking of participants' vocal responses, are provided as supplementary material. Raw vocal responses contain personally identifying information (i.e., the participants' voices) and therefore cannot be made publicly available. This data-sharing strategy complies with the requirements of the current study's funders and with the institutional ethics approval.

\section{Results}

Our subjects progressed significantly in tactile reading during their Braille training, reaching an average performance of 8.5 Braille words read per minute (WPM; $\mathrm{SD}=4.2 \mathrm{WPM}$; range $=3-16 \mathrm{WPM}$ ) and 17.3 Braille letters read per minute (LPM; SD = 4.2 LPM; range = 11-29 LPM) (see Supplementary Information for detailed behavioral results related to the Braille training). In the TMS experiment itself, the overall Braille letter recognition accuracy was $83 \%(\mathrm{SD}=12 \%$; range $=57-96 \%)$ and the overall reaction time was $1907 \mathrm{~ms}$ per Braille letter $(S D=522 \mathrm{~ms}$; range $=927-3051 \mathrm{~ms}$ ). 


\section{Accuracy - comparisons between TMS time windows}

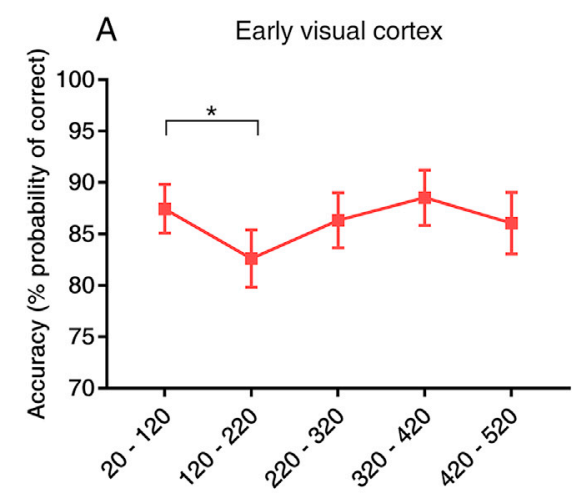

B
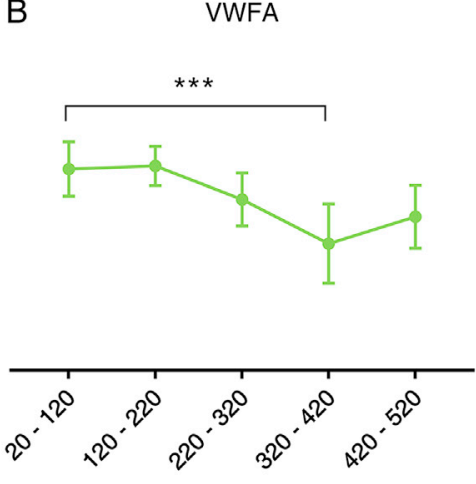

TMS time windows (ms)
C Early somatosensory cortex
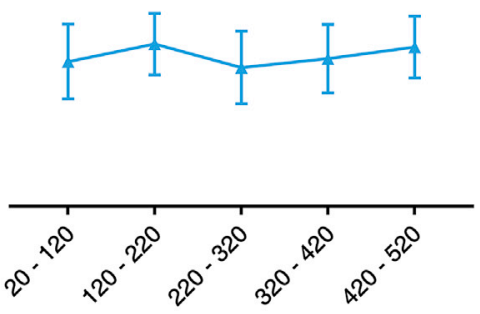

Fig. 2. Results of the Braille letter recognition accuracy analysis-comparisons within each TMS site. Comparisons between the earliest TMS time window and every other time window were performed for (A) the left early visual cortex stimulation; (B) the visual word form area (VWFA) stimulation and (C) the left early somatosensory cortex stimulation. The analysis was performed on marginal means reflecting the probability of a subject's correct recognition of a Braille letter under a given condition, which were estimated using a generalized linear mixed model (GLMM). * $\mathrm{p}<0.05,{ }^{* * *} \mathrm{p}=0.001$, Bonferroni-corrected for multiple comparisons. Error bars represent the standard error of the mean.

\section{Accuracy - comparisons between areas}

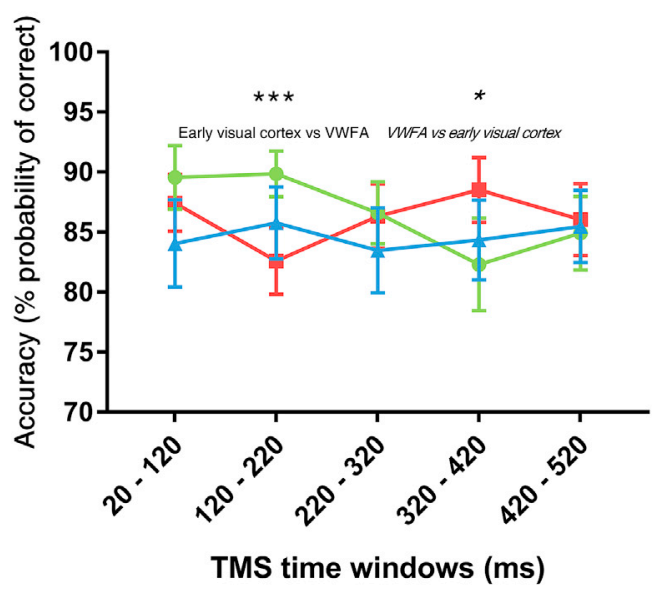

Early Visual Cortex $-\bullet$ VWFA $\_$Early Somatosensory Cortex

Fig. 3. Results of the Braille letter recognition accuracy analysis—comparisons between TMS sites. Asterisks indicate significant accuracy differences between the two TMS sites in a given time window. ${ }^{*} \mathrm{p}<0.05,{ }^{* * *} \mathrm{p}=0.001$, Bonferronicorrected for multiple comparisons. Error bars represent the standard error of the mean.

\subsection{TMS effects on braille letter recognition accuracy}

The GLMM applied to the analysis of the accuracy data (see Section 2.9; see also Jaeger, 2008) correctly classified $83 \%$ of all subjects' responses. The corrected model (i.e., including all independent variables; see Section 2.9) classified the data better than did the null model (which solely included an intercept; $\left.\mathrm{F}_{(14,5084)}=11.2, p<0.001\right)$. In the corrected model, no significant main effects were detected for either the TMS site $\left(\mathrm{F}_{(2,5084)}=1.2, p=0.311\right)$ or the TMS time window $\left(\mathrm{F}_{(4,}\right.$, $5084)=1.4, p=0.236$ ), although we observed a significant TMS site $\mathrm{x}$ TMS time window interaction $\left(\mathrm{F}_{(8,5084)}=13.3, p<0.001\right)$. Fixed coefficients for the GLMM are presented in Supplementary Table S1. The random effect included in the model (reflecting between-subject variability; see Section 2.9) was also significant with the intercept of 0.74 $(\mathrm{SE}=0.28$; Wald $\mathrm{Z}=2.71, p=0.007)$.

Pairwise comparisons within each TMS site revealed that, relative to the earliest TMS time window $(20-120 \mathrm{~ms})$, the probability of recognizing a Braille letter correctly decreased when TMS was applied at the $120-220 \mathrm{~ms}$ time window to the early visual cortex $\left(\mathrm{t}_{(5084)}=2.97, p_{\text {un- }}\right.$ corr $=0.003, p_{\text {corr }}=0.012$; Fig. $2 \mathrm{~A}$ ) and at the $320-420 \mathrm{~ms}$ time window to the VWFA $\left(\mathrm{t}_{(5084)}=3.52\right.$, $p_{\text {uncorr }}<0.001, p_{\text {corr }}=0.001$; Fig. $\left.2 \mathrm{~B}\right)$. Interestingly, no significant effects were detected for the early somatosensory cortex stimulation (all $p_{\text {corr }}>0.25$; Fig. $2 \mathrm{C}$ ).

Pairwise comparisons between TMS sites within each TMS time window revealed a significantly decreased probability that a subject would recognize a Braille letter correctly for the early visual cortex stimulation relative to the VWFA stimulation in the $120-220 \mathrm{~ms}$ TMS time window $\left(\mathrm{t}_{(5084)}=4.14, p_{\text {uncorr }}<0.001, p_{\text {corr }}<0.001\right.$; Fig. 3$)$. An inverse pattern was observed at the $320-420 \mathrm{~ms}$ time window, with the probability of a subject correctly recognizing a Braille letter being lower for the VWFA stimulation than for the early visual cortex stimulation $\left(\mathrm{t}_{(5084)}=2.49, p_{\text {uncorr }}=0.013, p_{\text {corr }}=0.039\right.$; Fig. 3). No significant differences were found in comparisons including the early somatosensory cortex or other TMS time windows (all $p_{\text {corr }}>0.08$; Fig. 3). Supplementary analyses suggest that detected decreases in the probability that a subject would provide a correct answer were largely driven by an increase in the probability that a subject would provide an incorrect answer rather than an increase in the probability of a missing response (see Supplementary Figs. S1-S2).

In line with the results obtained within the main model, in the GLMM that solely included the early visual cortex and the VWFA as TMS sites we detected a significant interaction between the TMS site and the TMS time window $\left(F_{(4,3389)}=13.7, p<0.001\right)$ and no significant main effects of either the TMS site $\left(\mathrm{F}_{(1,3389)}=0.2, p=0.652\right)$ or TMS time window $\left(\mathrm{F}_{(4}\right.$, $3389)=1.77, p=0.132$ ). In the pairwise comparisons, FDR-corrected across all tests performed within a model (i.e., thirteen tests; see Section 2.9), we replicated all effects obtained in the main analysis (Table 1).

\subsection{TMS effects on braille letter recognition speed}

The ANOVA for the reaction time data exhibited no significant TMS site $\mathrm{x}$ TMS time window interaction $\left(\mathrm{F}_{(8,128)}=1.12, p=0.357\right.$, $\left.\eta_{\mathrm{p}}^{2}=0.065\right)$. However, significant main effects of the TMS site $\left(\mathrm{F}_{(2}\right.$, 
RTs - comparisons between areas (TMS time windows combined)

A

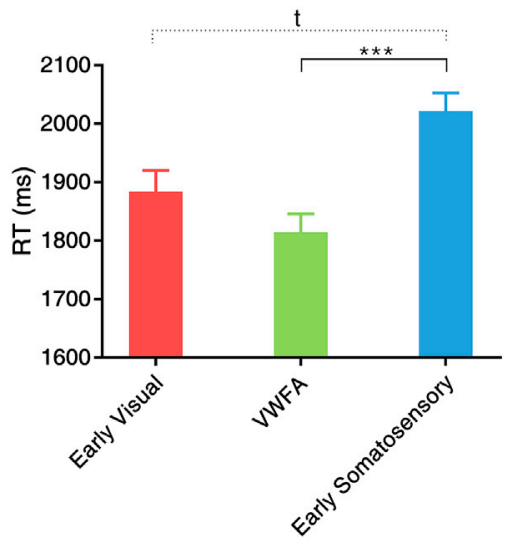

Stimulated area
RTs - comparisons between TMS time windows (TMS areas combined)

B

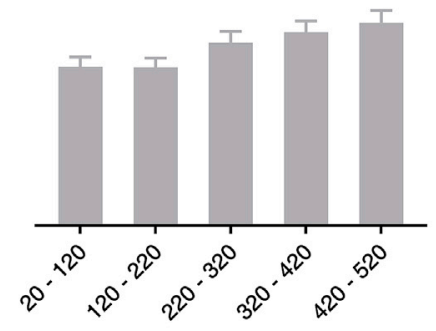

Fig. 4. Results of the Braille letter recognition speed analysis. To explore the significant main effects of a TMS site and a TMS time window on subjects' reaction times (represented as "RTs" in the images above), comparisons were performed between: (A) all TMS sites, with data from TMS time windows combined within each site; and (B) the first TMS time windows and every other time window, with data from all TMS sites combined within each TMS time window. ${ }^{* * * \mathrm{p}}=0.001,{ }^{\mathrm{t}} \mathrm{p}=0.051$, Bonferronicorrected for multiple comparisons. Error bars represent the standard error of the mean, adjusted to reflect between-subject variance in changes in reaction times across (A) TMS sites or (B) TMS time windows using a method proposed by Cousineau (2005). While the actual reaction time analysis was performed on the log-transformed data, reaction times prior to the log transformation are presented to enhance the figure's interpretability.
Table 1

Results of the Braille letter recognition accuracy analysis-pairwise comparisons including the early visual cortex and the VWFA, FDR-corrected for multiple comparisons. Significant results are bolded; TMS TW-TMS time window.

\begin{tabular}{|c|c|c|c|c|c|}
\hline Contrast & $\begin{array}{l}\text { Contrast } \\
\text { estimate }\end{array}$ & $\begin{array}{l}\text { Standard } \\
\text { error }\end{array}$ & $\begin{array}{l}\mathrm{t}- \\
\text { value }\end{array}$ & $\mathrm{p}$-value & $\begin{array}{l}\text { FDR- } \\
\text { adjusted p- } \\
\text { value }\end{array}$ \\
\hline $\begin{array}{l}\text { Early visual: } \\
\text { TMS TW } 1 \text { vs. } \\
\text { TMS TW } 2\end{array}$ & -0.048 & 0.016 & 2.97 & 0.003 & 0.013 \\
\hline $\begin{array}{l}\text { Early visual: } \\
\text { TMS TW } 1 \text { vs. } \\
\text { TMS TW } 3\end{array}$ & -0.011 & 0.021 & 0.52 & 0.604 & 0.785 \\
\hline $\begin{array}{l}\text { Early visual: } \\
\text { TMS TW } 1 \text { vs. } \\
\text { TMS TW } 4\end{array}$ & 0.011 & 0.017 & 0.65 & 0.519 & 0.75 \\
\hline $\begin{array}{l}\text { Early visual: } \\
\text { TMS TW } 1 \text { vs. } \\
\text { TMS TW } 5\end{array}$ & -0.014 & 0.017 & 0.81 & 0.419 & 0.681 \\
\hline $\begin{array}{l}\text { VWFA: TMS TW } \\
1 \text { vs. TMS TW } \\
2\end{array}$ & 0.003 & 0.016 & 0.18 & 0.855 & 0.901 \\
\hline $\begin{array}{l}\text { VWFA: TMS TW } \\
1 \text { vs. TMS TW } \\
3\end{array}$ & -0.030 & 0.020 & 1.51 & 0.130 & 0.282 \\
\hline $\begin{array}{l}\text { VWFA: TMS TW } \\
1 \text { vs. TMS TW } \\
4\end{array}$ & -0.072 & 0.021 & 3.53 & $<0.001$ & 0.003 \\
\hline $\begin{array}{l}\text { VWFA: TMS TW } \\
1 \text { vs. TMS TW } \\
5\end{array}$ & -0.046 & 0.025 & 1.88 & 0.06 & 0.156 \\
\hline $\begin{array}{l}\text { TMS TW 1: early } \\
\text { visual vs. } \\
\text { VWFA }\end{array}$ & 0.021 & 0.018 & 1.2 & 0.232 & 0.431 \\
\hline $\begin{array}{l}\text { TMS TW 2: } \\
\text { early visual } \\
\text { vs. VWFA }\end{array}$ & 0.72 & 0.018 & 4.11 & $<0.001$ & $<0.001$ \\
\hline $\begin{array}{l}\text { TMS TW 3: early } \\
\text { visual vs. } \\
\text { VWFA }\end{array}$ & 0.003 & 0.022 & 0.12 & 0.901 & 0.901 \\
\hline $\begin{array}{l}\text { TMS TW 4: } \\
\text { early visual } \\
\text { vs. VWFA }\end{array}$ & -0.062 & 0.025 & 2.5 & 0.012 & 0.039 \\
\hline $\begin{array}{l}\text { TMS TW 5: early } \\
\text { visual vs. } \\
\text { VWFA }\end{array}$ & -0.11 & 0.029 & 0.39 & 0.697 & 0.824 \\
\hline
\end{tabular}

32) $\left.=7.92, p=0.002, \eta_{\mathrm{p}}^{2}=0.331\right)$ and TMS time window $\left(\mathrm{F}_{(4,64)}=2.58\right.$, $\left.p=0.046, \eta_{\mathrm{p}}^{2}=0.139\right)$ were detected. Pairwise comparisons between TMS sites (Fig. 4A) revealed that reaction times were significantly greater when TMS was applied to the early somatosensory cortex rather than the
VWFA $\left(+208 \mathrm{~ms} ; \mathrm{t}_{(16)}=4.34, p_{\text {uncorr }}=0.001, p_{\text {corr }}=0.003 ; \mathrm{d}=0.35\right)$ and, at trend level of significance, when TMS was applied to the early somatosensory cortex rather than the early visual cortex $(+137 \mathrm{~ms}$; $\left.\mathrm{t}_{(16)}=2.67, p_{\text {uncorr }}=0.017, p_{\text {corr }}=0.051 ; \mathrm{d}=0.24\right)$. Pairwise comparisons between TMS time windows (Fig. 4B) did not reveal any significant differences in reaction times (the first time window vs. every other time window: all $p_{\text {corr }}>0.13$; Fig. 4B).

The ANOVA that solely included the early visual cortex and the VWFA as TMS sites did not reveal any significant effects (all $p>0.18$ ).

\section{Discussion}

In this study, we employed a chronometric TMS to demonstrate that the early and ventral visual cortices support tactile Braille letter recognition in sighted adults. We observed specific spatiotemporal dynamics of this cross-modal involvement; the early visual cortex was critically involved in the Braille letter recognition 120-220 ms following the letter presentation, whereas the VWFA was critical for this task $320-420 \mathrm{~ms}$ following the letter presentation. These results indicate that sighted people's visual cortices are involved in tactile perception in a hierarchical manner. Furthermore, our findings suggest that this cross-modal involvement respects the canonical visual processing hierarchy; early stages of tactile processing are supported by the early visual cortex, whereas more advanced tactile computations involve high-level visual areas.

Our results align with previous studies that have documented crossmodal activations at the various visual processing hierarchy stages in sighted people. Some of these responses emerge as a result of learning new tactile or auditory skills (Amedi et al., 2007; Saito et al., 2006; Siuda-Krzywicka et al., 2016; Zangenehpour and Zatorre, 2010), while others are observed without any specific training, thus suggesting they are part of a functional repertoire allowed by the default cortical organization (Amedi et al., 2001; Campus et al., 2017; Eck et al., 2016, 2013; Lacey et al., 2014, 2010; Sathian et al., 2011; Stilla and Sathian, 2008; Tal et al., 2016). In the context of our work, it is particularly interesting that the fMRI study of Snow et al. (2014) has already suggested that neural populations responsive to tactually perceived shapes exist both in the early visual cortices and in higher-level ventral visual regions (i.e., V4 and the lateral occipital complex) of sighted subjects. Our results advance this finding by demonstrating that early and ventral visual cortices' involvement in tactile perception is hierarchical and functionally relevant. The fact that the visual cortex's stimulation disrupted tactile letter recognition in sighted subjects-despite their not having been blindfolded-raises the possibility that cross-modal interactions between the 
tactile and visual system occur in many everyday situations.

We believe our results contribute to a discussion concerning the extent to which the brain can be viewed as "metamodal"-that is, composed of areas showing preference for specific computations independently of sensory input modality (Amedi et al., 2017; Pascual-Leone and Hamilton, 2001). The metamodal account of brain organization has recently received considerable support from research on blind and deaf individuals (Amedi et al., 2017; Benetti et al., 2017; Bola et al., 2017c; Heimler et al., 2015; Lomber et al., 2010; Meredith et al., 2011; for counter-arguments, see Bedny, 2017). Several studies suggest that a metamodal account can be also applied to the non-deprived brain; for example, the lateral occipital complex, which is strongly activated during visual object recognition (Malach et al., 1995), is also preferentially recruited for tactile and auditory object recognition (Amedi et al., 2007, 2001; Kim and Zatorre, 2011; Lacey et al., 2014, 2010), whereas the VWFA, an area that develops functional preference for visual words and letters (Dehaene and Cohen, 2011; Price and Devlin, 2011), is strongly activated by tactile Braille reading (Siuda-Krzywicka et al., 2016). Similarly, the V5/MT area, which exhibits functional preference for moving visual stimuli (Zeki et al., 1991), becomes activated by dynamic tactile and auditory stimuli (Hagen et al., 2002; Poirier et al., 2005). While most of these studies focus on the high-level visual cortex, one recent work (Campus et al., 2017) demonstrates that the early visual cortex can be recruited for spatial although not temporal auditory processing, thus increasing the possibility that the metamodal principle can be applied to low-level visual cortices to some extent. By demonstrating that the early visual cortex was causally involved in a specific early stage of tactile letter recognition, our results further support this possibility. Moreover, our work supports the idea of the visual cortex's metamodal organization from a more general perspective of the propagation of cross-modal information in this cortical system. While previous studies have primarily focused on specific visual regions, our findings suggest that early and high-level visual cortices can be gradually engaged in tactile processing depending upon the task at hand's current computational demands.

Based on the metamodal account of the visual system's organization, we hypothesized that, during tactile Braille reading, the early visual cortex participates in the construction of a spatial representation of Braille dots and signs, whereas the VWFA supports the creation of an abstract representation of a Braille letter. In line with this proposal, previous TMS studies have shown that sighted people's early visual cortex supports spatial, non-linguistic tasks performed in the tactile modality, such as discrimination of Braille signs' shapes (same/different decision; Merabet et al., 2008), discrimination of tactually presented gratings' orientations (Zangaladze et al., 1999), and distance judgments performed on Braille-like dots (Merabet et al., 2004). Furthermore, fMRI experiments documented the early visual cortex's recruitment for tactile texture perception (Eck et al., 2016, 2013; Sathian et al., 2011; Stilla and Sathian, 2008). The VWFA's role in tactile perception was investigated in our previous study (Siuda-Krzywicka et al., 2016), wherein we demonstrated that, relative to touching strings of nonsense Braille characters, whole-word tactile Braille reading activates this cortical region in sighted subjects and that TMS applied to sighted subjects' VWFA disrupts performance in a tactile lexical decision task similarly to the way it disrupts performance in a visual lexical decision task performed in the Latin alphabet (as reported by Duncan et al., 2010). To our knowledge, no study on sighted subjects has proven this cortical area's involvement in tactile tasks that do not involve recognizing letters or words, which suggests that the VWFA's cross-modal involvement might be specific to this cognitive domain. The present results seem to generally agree with the available literature as well as our initial hypothesis regarding the division of labor between the early visual cortex and the VWFA. Notably, the early visual cortex's involvement in the tactile recognition of Braille signs was observed relatively quickly (i.e., 120-220 ms following the Braille letter presentation's onset). This is particularly interesting given the fact that tactile recognition is usually much slower than visual recognition (see, e.g., Kitada et al., 2014). Moreover, our subjects' tactile letter and word reading speeds were massively slower than the typical visual reading speeds of sighted people (around 200-250 WPM; Hunziker, 2006) or even the typical Braille reading speeds of blind people (Legge et al., 1999). Several studies have demonstrated that TMS applied to the early visual cortex at comparable time windows disrupts non-linguistic processing in the visual (Koivisto et al., 2011; Koivisto and Silvanto, 2012) and tactile modality (Zangaladze et al., 1999), which supports our hypothesis regarding the early visual cortex's relatively basic, spatial role in tactile reading. Only significantly later (320-420 ms following the Braille letter presentation's onset) did the Braille letter recognition involve the VWFA, which is suggestive of this area's qualitatively different role in this task.

The identification of specific neural mechanisms underlying the visual cortex's involvement in tactile perception remains a field of intensive inquiry. From a theoretical perspective, two broad families of processes should be considered: (1) "bottom-up" mechanisms, which directly map certain kinds of tactile information onto the visual cortex processing machinery, and (2) indirect "top-down" mechanisms, which take the form of either conscious visual imagery or unconscious feedback signals propagating from higher-level cortical regions to the visual cortex. In recent years, empirical evidence was provided in support of both these possibilities (see, e.g., Amedi et al., 2001; Deshpande et al., 2010; Lacey et al., 2014, 2010; Merabet et al., 2006; Siuda-Krzywicka et al., 2016). As proposed by Lacey et al. (2009), these two types of mechanisms may very well act in concert, and their relative contributions (and an exact type of top-down influences) to the visual cortex's involvement in tactile perception might to some extent depend upon a subject's familiarity with an object that is touched. This theoretical proposal was largely confirmed in a series of studies concerning the role of the lateral occipital complex (LOC) in tactile shape processing. The authors revealed that, during a tactile exploration of familiar objects, the LOC activation's magnitude was correlated with the magnitude of activation evoked in this area by a visual object imagery condition (Lacey et al., 2010). Moreover, an effective connectivity analysis indicated that, under both these conditions, the LOC's activation was primarily driven by inputs from the prefrontal cortex (Deshpande et al., 2010). In contrast, the LOC activation's magnitude during the tactile exploration of unfamiliar objects was not correlated with the magnitude of activation evoked in this region by either visual object imagery or spatial imagery (Lacey et al., 2014, 2010). Furthermore, the LOC's activation during the tactile exploration of unfamiliar objects was primarily driven by inputs from the somatosensory system and the intraparietal sulcus (Deshpande et al., 2010; Lacey et al., 2014). The authors concluded that the LOC hosts a modality-independent representation of an object's shape, which can be accessed both by bottom-up and top-down mechanisms.

We believe our results might contribute to the development of the above-described model in several ways. Firstly, we revealed that the ventral visual stream's involvement in tactile perception can be preceded by the involvement of the visual processing hierarchy's earlier stages. Based on our results, we cannot establish whether the information computed in the early visual cortex during Braille letter recognition is then transferred to the VWFA or whether the information processed in these two regions is integrated outside the visual cortex. Nevertheless, our study raises the possibility that inputs from early visual cortices are another important driver of high-level visual areas' involvement in tactile perception - a hypothesis that, to our knowledge, has not yet been directly tested. Secondly, our results suggest that mechanisms of the visual cortex's involvement in the same tactile task might significantly vary as a function of time following the stimulus presentation, thus suggesting that this parameter should perhaps be included in the model. Based on a distinction between the tactile perception of familiar and unfamiliar objects made by Lacey et al. (2009), one might specifically expect to observe a stronger contribution of bottom-up mechanisms to the visual cortex's cross-modal involvement at the onset of a subject's interactions with a tactile object, when information about its shape and identity is 
limited; in contrast, top-down mechanisms should gain importance with time. Finally, based on these considerations, one might also expect that the bottom-up mechanisms' contribution is more pronounced in the case of early visual areas, which seem to be engaged in tactile perception at earlier time windows than are high-level visual areas. Overall, our study reveals that the visual cortex's cross-modal involvement is a dynamic process that develops within both space and time.

The subjects recruited for this study were able to visually read Braille signs even prior to the initiation of their tactile Braille reading training. During their training, the subjects often visually checked Braille exercises performed in the tactile modality, which perhaps explains why they improved their visual Braille reading speed following our study (see Supplementary Information for behavioral results related to the tactile Braille training; for details regarding how the tactile Braille training was designed, see Bola et al., 2016). This result might raise a question regarding whether their visual familiarity with Braille signs may have influenced the results of our TMS experiment. From the theoretical standpoint, studying tactile perception without any form of contamination by the visual experience with the object that is touched is perhaps exclusively possible when congenitally blind subjects who have not developed the visual imagery mechanism are studied; arguably, every sighted person would instantly start to imagine a tactually explored object, which should likely be treated as a form of visual experience even if triggered internally. Nevertheless, one might specifically wonder whether the subjects' training in the visual Braille reading-a visual counterpart of the tactile task they performed in the present experiment-may have influenced the mechanisms of the visual cortex's cross-modal involvement that we have reported herein. Within the framework of the model developed by Lacey et al. (2009) that was described above, it seems likely that prior training in visual Braille reading amplifies the process of the visual cortex's cross-modal involvement in tactile Braille reading observed in our study-perhaps by priming neuronal populations in the visual cortex with Braille-like shapes or by making the conceptual representation of the Braille script more salient and thereby increasing the strength of top-down signals reaching the visual cortex during tactile Braille reading. However, unless one assumes that learning to visually recognize Braille signs leads to the emergence of neuronal populations responsive to the shape of Braille dots or signs in the visual cortex, which then take over the processing of the same shapes conveyed by the tactile modality-a possibility we consider highly unlikely, especially given that our subjects were adults and their prior training in visual Braille reading constituted a very small portion of their overall visual experience-there exists no reason to believe that the visual Braille training qualitatively changes mechanisms of the visual cortex's cross-modal involvement in the process of reading this script tactually. In summary, we would expect to find similar (possibly less pronounced) effects even in subjects with no formal visual Braille training. Consistent with this line of reasoning, a considerable number of studies have already indicated that the visual cortex is involved in various forms of tactile perception (including the perception of Braille and Braille-like stimuli), even in subjects without any specific visual experience with objects they have touched (for studies including Braille and Braille-like shapes, see, e.g., Debowska et al., 2016; Merabet et al., 2008, 2004).

Finally, contrary to the visual system stimulation, we found that TMS applied to the early somatosensory cortex did not have any specific influence on the accuracy of our subjects' Braille letter recognition; rather, the early somatosensory cortex stimulation induced a general slowdown of subjects' reaction times independently of the TMS time window. Such a pattern of results is unexpected; based on the early somatosensory cortex's location in the tactile processing hierarchy, one might rather expect that a disruptive effect of TMS applied to this area would be present in the earliest time window and vanish in later time windows. Indeed, Zangaladze et al. (1999) demonstrated that the TMS of the early somatosensory cortex, applied $30 \mathrm{~ms}$ following the tactile presentation of a grating strongly disrupts the accuracy of judgments on its orientation. Our results might suggest that effective tactile perception critically depends upon the early somatosensory cortex's involvement even at much later time points-a possibility that seems counterintuitive although, to our knowledge, has not yet been specifically tested. It is important to stress, however, that any interpretation of the effect found for the early somatosensory cortex stimulation in our study should be made with caution, as our design was optimized for the detection of time-specific effects and thus lacks optimal control conditions for probing TMS effects present at all time windows. Notably, a recent study conducted by Holmes et al. (2019) argues that localizing the somatosensory hand area by moving a TMS coil posteriorly from the motor hand area is far from optimal. In that paper, the authors defined the motor hand area as a site at which TMS induces the strongest hand muscle response. In contrast, the localization of both the hand motor area and the early somatosensory site in our study was constrained by anatomical landmarks (see Section 2.4), which allowed us to avoid bias related to the imprecise localization of the "omega knob"- a problem that seems to be common among studies that employ the hand muscle response as an indicator of this region's location (see Ahdab et al., 2016) —and ensure that we localized the early somatosensory cortex within the postcentral gyrus. Nevertheless, given that the interindividual variability in the somatosensory hand area's location within the postcentral gyrus seems considerable (Holmes et al., 2019; Merzenich et al., 1987 Geyer et al., 1999; Grefkes et al., 2001; Schweisfurth et al., 2018), we cannot fully exclude the possibility that our method of localizing the early somatosensory cortex lacked the precision necessary to detect a true time-specific effect of neural activity disruption in this area, and that the observed general slowdown of reaction times reflects some confounds for which comparisons with other TMS sites cannot account. This concern does not apply, however, to our key results of the visual cortex stimulation, which are controlled across both TMS time windows and TMS sites.

In conclusion, we provide causal evidence that, in sighted adults, tactile Braille letter recognition is supported by the early visual and ventral visual cortices. Moreover, our results indicate that, in sighted people, the visual cortex's involvement in tactile perception respects the canonical visual hierarchy-that is, the early stages of tactile processing involve the early visual cortex whereas more advanced tactile computations involve high-level visual areas. In combination with our previous studies (Bola et al., 2017a; Siuda-Krzywicka et al., 2016), these findings reveal the visual cortex's remarkable multimodal potential to support tactile perception even when the visual input is unconstrained.

\section{Author contributions}

Ł.B., J.M., M.S., M.W.S., M.P., K.J., M.S. and A.M. designed research; Ł.B. and J.M performed research; Ł.B., J.M., M.S. and D.D. analyzed data; Ł.B., J.M., K.J., M.S. and A.M wrote the paper.

\section{Conflicts of interest}

The authors declare that no competing interests exist.

\section{Acknowledgements}

Supported by a National Science Centre Poland grant (2014/14/M/ HS6/00918) awarded to A.M., a National Science Centre Poland grant (2016/21/B/HS6/03703) awarded to M.S., a National Science Centre Poland grant (2014/15/N/HS6/04184) awarded to Ł.B, and a National Science Centre Poland grant (2017/27/N/HS6/02669) awarded to J.M. The study was conducted with the aid of CePT research infrastructure purchased with funds from the European Regional Development Fund as part of the Innovative Economy Operational Programme, 2007-2013.

\section{Appendix A. Supplementary data}

Supplementary data to this article can be found online at https://doi. org/10.1016/j.neuroimage.2019.116084. 


\section{References}

Ahdab, R., Ayache, S.S., Brugières, P., Farhat, W.H., Lefaucheur, J.P., 2016. The hand motor hotspot is not always located in the hand knob: a neuronavigated transcranial magnetic stimulation study. Brain Topogr. 29, 590-597.

Amassian, V.E., Cracco, R.Q., Maccabee, P.J., Cracco, J.B., Rudell, A., Eberle, L., 1989. Suppression of visual perception by magnetic coil stimulation of human occipital cortex. Electroencephalogr. Clin. Neurophysiol. 74, 458-462.

Amedi, A., Hofstetter, S., Maidenbaum, S., Heimler, B., 2017. Task selectivity as a comprehensive principle for brain organization. Trends Cogn. Sci. 21, 307-310.

Amedi, A., Malach, R., Hendler, T., Peled, S., Zohary, E., 2001. Visuo-haptic object-related activation in the ventral visual pathway. Nat. Neurosci. 4, 324-330.

Amedi, A., Stern, W.M., Camprodon, J.A., Bermpohl, F., Merabet, L., Rotman, S., Hemond, C., Meijer, P., Pascual-Leone, A., 2007. Shape conveyed by visual-toauditory sensory substitution activates the lateral occipital complex. Nat. Neurosci. 10, 687-689.

Amemiya, T., Beck, B., Walsh, V., Gomi, H., Haggard, P., 2017. Visual area V5/hMT+ contributes to perception of tactile motion direction: a TMS study. Sci. Rep. 7, 40937.

Argyropoulos, V., Papadimitriou, V., 2015. Braille reading accuracy of students who are visually impaired: the effects of gender, age at vision loss, and level of education. J. Vis. Impair. Blind. (JVIB) 109, 107-118.

Bavelier, D., Neville, H.J., 2002. Cross-modal plasticity: where and how? Nat. Rev. Neurosci. 3, 443-452.

Bedny, M., 2017. Evidence from blindness for a cognitively pluripotent cortex. Trends Cogn. Sci. 21, 637-648.

Benetti, S., van Ackeren, M.J., Rabini, G., Zonca, J., Foa, V., Baruffaldi, F., Rezk, M., Pavani, F., Rossion, B., Collignon, O., 2017. Functional selectivity for face processing in the temporal voice area of early deaf individuals. Proc. Natl. Acad. Sci. U. S. A 114, E6437-E6446.

Benjamini, Y., Hochberg, Y., 1995. Controlling the false discovery rate: a practical and powerful approach to multiple testing. J. R. Stat. Soc. Ser. B 57, 289-300.

Bola, Ł., Siuda-Krzywicka, K., Paplińska, M., Sumera, E., Hańczur, P., Szwed, M., 2016. Braille in the sighted: teaching tactile reading to sighted adults. PLoS One 11, e0155394.

Bola, Ł., Siuda-Krzywicka, K., Paplińska, M., Sumera, E., Zimmermann, M., Jednoróg, K., Marchewka, A., Szwed, M., 2017a. Structural reorganization of the early visual cortex following Braille training in sighted adults. Sci. Rep. 7, 17448.

Bola, Ł., Radziun, D., Siuda-Krzywicka, K., Sowa, J.E., Paplińska, M., Sumera, E., Szwed, M., 2017b. Universal visual features might be necessary for fluent reading. A longitudinal study of visual reading in Braille and Cyrillic alphabets. Front. Psychol. 8, 514 .

Bola, Ł., Zimmermann, M., Mostowski, P., Jednoróg, K., Marchewka, A., Rutkowski, P. Szwed, M., 2017c. Task-specific reorganization of the auditory cortex in deaf humans. Proc. Natl. Acad. Sci. 114, E600-E609.

Campus, C., Sandini, G., Concetta Morrone, M., Gori, M., 2017. Spatial localization of sound elicits early responses from occipital visual cortex in humans. Sci. Rep. 7 , 10415.

Chambers, C.D., Allen, C.P.G., Maizey, L., Williams, M.A., 2013. Is delayed foveal feedback critical for extra-foveal perception? Cortex 49, 327-335.

Cohen, L., Lehéricy, S., Chochon, F., Lemer, C., Rivaud, S., Dehaene, S., 2002. Languagespecific tuning of visual cortex? Functional properties of the visual word form area. Brain 125, 1054-1069.

Cousineau, D., 2005. Confidence intervals in within-subject designs: a simpler solution to Loftus and Masson's method. Tutorials Quant. Methods Psychol. 1, 42-45.

De Graaf, T.A., Sack, A.T., 2011. Null results in TMS: from absence of evidence to evidence of absence. Neurosci. Biobehav. Rev. 35, 871-877.

Debowska, W., Wolak, T., Nowicka, A., Kozak, A., Szwed, M., Kossut, M., 2016. Functional and structural neuroplasticity induced by short-term tactile training based on Braille reading. Front. Neurosci. 10, 460.

Dehaene, S., Cohen, L., 2011. The unique role of the visual word form area in reading. Trends Cogn. Sci. 15, 254-262.

Dehaene, S., Cohen, L., Sigman, M., Vinckier, F., 2005. The neural code for written words: a proposal. Trends Cogn. Sci. 9, 335-341.

Deshpande, G., Hu, X., Lacey, S., Stilla, R., Sathian, K., 2010. Object familiarity modulates effective connectivity during haptic shape perception. Neuroimage 49, 1991-2000.

Duncan, K., Pattamadilok, C., Devlin, J., 2010. Investigating occipito-temporal contributions to reading with TMS. J. Cogn. Neurosci. 22, 739-750.

Eck, J., Kaas, A.L., Goebel, R., 2013. Crossmodal interactions of haptic and visual texture information in early sensory cortex. Neuroimage 75, 123-135.

Eck, J., Kaas, A.L., Mulders, J.L., Hausfeld, L., Kourtzi, Z., Goebel, R., 2016. The effect of task instruction on haptic texture processing: the neural underpinning of roughness and spatial density perception. Cerebr. Cortex 26, 384-401.

Glezer, L.S., Jiang, X., Riesenhuber, M., 2009. Evidence for highly selective neuronal tuning to whole words in the "visual word form area". Neuron 62, 199-204.

Geyer, S., Schleicher, A., Zilles, K., 1999. Areas 3a, 3b, and 1 of human primary somatosensory cortex: 1 . Microstructural organization and interindividual variability. Neuroimage 10, 63-83.

Grefkes, C., Geyer, S., Schormann, T., Roland, P., Zilles, K., 2001. Human somatosensory area 2: observer-independent cytoarchitectonic mapping, interindividual variability, and population map. Neuroimage 14, 617-631.

Hagen, M.C., Franzén, O., McGlone, F., Essick, G., Dancer, C., Pardo, J.V., 2002. Tactile motion activates the human middle temporal/V5 (MT/V5) complex. Eur. J. Neurosci. 16, 957-964.

Heimler, B., Striem-Amit, E., Amedi, A., 2015. Origins of task-specific sensoryindependent organization in the visual and auditory brain: neuroscience evidence, open questions and clinical implications. Curr. Opin. Neurobiol. 35, 169-177.
Holmes, N.P., Tamè, L., Beeching, P., Medford, M., Rakova, M., Stuart, A., Zeni, S., 2019. Locating primary somatosensory cortex in human brain stimulation studies: experimental evidence. J. Neurophysiol. 121, 336-344.

Hunziker, H.W., 2006. Im Auge des Lesers: foveale und periphere Wahrnehmung - vom Buchstabieren zur Lesefreude [The eye of the reader: foveal and peripheral perception - from letter recognition to the joy of reading. Transmedia, Zurich.

Jaeger, T.F., 2008. Categorical data analysis: away from ANOVAs (transformation or not) and towards logit mixed models. J. Mem. Lang. 59, 434-446.

Kandel, E., Schwartz, J., Jessell, T., Siegelbaum, S.A., Hudspeth, A.J., 2012. Principles of Neural Science. McGraw-Hill, New York.

Kim, J.-K., Zatorre, R.J., 2011. Tactile-auditory shape learning engages the lateral occipital complex. J. Neurosci. 31, 7848-7856.

Kitada, R., Yoshihara, K., Sasaki, A.T., Hashiguchi, M., Kochiyama, T., Sadato, N., 2014. The brain network underlying the recognition of hand gestures in the blind: the supramodal role of the extrastriate body area. J. Neurosci. 34, 10096-10108.

Koivisto, M., Railo, H., Revonsuo, A., Vanni, S., Salminen-Vaparanta, N., 2011. Recurrent processing in V1/V2 contributes to categorization of natural scenes. J. Neurosci. 31, 2488-2492.

Koivisto, M., Silvanto, J., 2012. Visual feature binding: the critical time windows of V1/ V2 and parietal activity. Neuroimage 59, 1608-1614.

Konopnicki, J., 1961. Problem opóźnienia w nauce szkolnej [Problem of delay in school education]. Zakład Narodowy im. Ossolińskich Wydawnictwo PAN.

Lacey, S., Flueckiger, P., Stilla, R., Lava, M., Sathian, K., 2010. Object familiarity modulates the relationship between visual object imagery and haptic shape perception. Neuroimage 49, 1977-1990.

Lacey, S., Stilla, R., Sreenivasan, K., Deshpande, G., Sathian, K., 2014. Spatial imagery in haptic shape perception. Neuropsychologia 60, 144-158.

Lacey, S., Tal, N., Amedi, A., Sathian, K., 2009. A putative model of multisensory object representation. Brain Topogr. 21, 269-274, 009-0087-4.

Legge, G.E., Madison, C.M., Mansfield, J.S., 1999. Measuring Braille reading speed with the MNREAD test. Vis. Impair. Res. 1, 131-145.

Lomber, S.G., Meredith, M.A., Kral, A., 2011. Adaptive crossmodal plasticity in deaf auditory cortex: areal and laminar contributions to supranormal vision in the deaf. Prog. Brain Res. 191, 251-270.

Lomber, S.G., Meredith, M.A., Kral, A., 2010. Cross-modal plasticity in specific auditory cortices underlies visual compensations in the deaf. Nat. Neurosci. 13, 1421-1427.

Malach, R., Reppas, J.B., Benson, R.R., Kwong, K.K., Jiang, H., Kennedy, W.A. Ledden, P.J., Brady, T.J., Rosen, B.R., Tootell, R.B., 1995. Object-related activity revealed by functional magnetic resonance imaging in human occipital cortex. Proc. Natl. Acad. Sci. 92, 8135-8139.

McDonald, J.H., 2009. Handbook of Biological Statistics. Sparky House Publishing.

Merabet, L.B., Hamilton, R., Schlaug, G., Swisher, J.D., Kiriakopoulos, E.T., Pitskel, N.B., Kauffman, T., Pascual-Leone, A., 2008. Rapid and reversible recruitment of early visual cortex for touch. PLoS One 3, e3046.

Merabet, L.B., Pascual-Leone, A., 2010. Neural reorganization following sensory loss: the opportunity of change. Nat. Rev. Neurosci. 11, 44-52.

Merabet, L.B., Swisher, J.D., McMains, S.A., Halko, M.A., Amedi, A., Pascual-Leone, A. Somers, D.C., 2006. Combined activation and deactivation of visual cortex during tactile sensory processing. J. Neurophysiol. 97, 1633-1641.

Merabet, L.B., Thut, G., Murray, B., Andrews, J., Hsiao, S., Pascual-Leone, A., 2004. Feeling by sight or seeing by touch? Neuron 42, 173-179.

Meredith, M.A., Kryklywy, J., McMillan, A.J., Malhotra, S., Lum-Tai, R., Lomber, S.G., 2011. Crossmodal reorganization in the early deaf switches sensory, but not behavioral roles of auditory cortex. Proc. Natl. Acad. Sci. U. S. A 108, 8856-8861.

Merzenich, M.M., Nelson, R.J., Kaas, J.H., Stryker, M.P., Jenkins, W.M., Zook, J.M., Cynader, M.S., Schoppmann, A., 1987. Variability in hand surface representations in areas 3b and 1 in adult owl and squirrel monkeys. J. Comp. Neurol. 258, 281-296.

Pascual-Leone, A., Hamilton, R., 2001. The metamodal organization of the brain. Prog. Brain Res. 134, 427-445.

Pascual-Leone, A., Walsh, V., Rothwell, J., 2000. Transcranial magnetic stimulation in cognitive neuroscience - virtual lesion, chronometry, and functional connectivity. Curr. Opin. Neurobiol. 10, 232-237.

Pattamadilok, C., Bulnes, L.C., Devlin, J.T., Bourguignon, M., Morais, J., Goldman, S., Kolinsky, R., 2015. How early does the brain distinguish between regular words, irregular words, and pseudowords during the reading process? Evidence from neurochronometric TMS. J. Cogn. Neurosci. 27, 1259-1274.

Pattamadilok, C., Knierim, I.N., Kawabata Duncan, K.J., Devlin, J.T., 2010. How does learning to read affect speech perception? J. Neurosci. 30, 8435-8444.

Peirce, J.W., 2007. PsychoPy-Psychophysics software in Python. J. Neurosci. Methods $162,8-13$.

Poirier, C., Collignon, O., DeVolder, A.G., Renier, L., Vanlierde, A., Tranduy, D., Scheiber, C., 2005. Specific activation of the V5 brain area by auditory motion processing: an fMRI study. Cogn. Brain Res. 25, 650-658.

Price, C.J., Devlin, J.T., 2011. The interactive account of ventral occipitotemporal contributions to reading. Trends Cogn. Sci. 15, 246-253.

Rauschecker, A.M., Bowen, R.F., Perry, L.M., Kevan, A.M., Dougherty, R.F., Wandell, B.A., 2011. Visual feature-tolerance in the reading network. Neuron 71, 941-953.

Rauschecker, J.P., 1995. Compensatory plasticity and sensory substitution in the cerebral cortex. Trends Neurosci. 18, 36-43.

Reddy, L., Kanwisher, N., 2006. Coding of visual objects in the ventral stream. Curr. Opin. Neurobiol. 16, 408-414.

Riesenhuber, M., Poggio, T., 2000. Models of object recognition. Nat. Neurosci. 3, 1199-1204.

Rolls, E.T., 2001. Functions of the primate temporal lobe cortical visual areas in invariant visual object and face recognition. In: Vision: the Approach of Biophysics and Neurosciences, pp. 366-395. 
Sack, A.T., Camprodon, J.A., Pascual-Leone, A., Goebel, R., 2005. The dynamics of interhemispheric compensatory processes in mental imagery. Science 308, 702-704.

Saito, D.N., Okada, T., Honda, M., Yonekura, Y., Sadato, N., 2006. Practice makes perfect: the neural substrates of tactile discrimination by Mah-Jong experts include the primary visual cortex. BMC Neurosci. 7, 79.

Salminen-Vaparanta, N., Koivisto, M., Noreika, V., Vanni, S., Revonsuo, A., 2012. Neuronavigated transcranial magnetic stimulation suggests that area V2 is necessary for visual awareness. Neuropsychologia 50, 1621-1627.

Sathian, K., Lacey, S., Stilla, R., Gibson, G.O., Deshpande, G., Hu, X., LaConte, S., Glielmi, C., 2011. Dual pathways for haptic and visual perception of spatial and texture information. Neuroimage 57, 462-475.

Sathian, K., Zangaladze, A., Hoffman, J.M., Grafton, S.T., 1997. Feeling with the mind's eye. Neuroreport 8, 3877-3881.

Schuhmann, T., Schiller, N.O., Goebel, R., Sack, A.T., 2012. Speaking of which: dissecting the neurocognitive network of language production in picture naming. Cerebr. Cortex 22, 701-709.

Schweisfurth, M.A., Frahm, J., Farina, D., Schweizer, R., 2018. Comparison of fMRI digit representations of the dominant and non-dominant hand in the human primary somatosensory cortex. Front. Hum. Neurosci. 12.

Siuda-Krzywicka, K., Bola, Ł., Paplińska, M., Sumera, E., Jednoróg, K., Marchewka, A., Śliwińska, M.W., Amedi, A., Szwed, M., 2016. Massive cortical reorganization in sighted Braille readers. Elife 5, e10762.

Sliwinska, M.W., James, A., Devlin, J.T., 2015. Inferior parietal lobule contributions to visual word recognition. J. Cogn. Neurosci. 27, 593-604.
Sliwinska, M.W., Khadilkar, M., Campbell-Ratcliffe, J., Quevenco, F., Devlin, J.T., 2012. Early and sustained supramarginal gyrus contributions to phonological processing. Front. Psychol. 3, 161.

Snow, J.C., Strother, L., Humphreys, G.W., 2014. Haptic shape processing in visual cortex. J. Cogn. Neurosci. 26, 1154-1167.

Stilla, R., Sathian, K., 2008. Selective visuo-haptic processing of shape and texture. Hum. Brain Mapp. 29, 1123-1138.

Sur, M., Pallas, S., Roe, A., 1990. Cross-modal plasticity in cortical development: differentiation and specification of sensory neocortex. Trends Neurosci. 13, 227-233.

Tal, Z., Geva, R., Amedi, A., 2016. The origins of metamodality in visual object area LO: bodily topographical biases and increased functional connectivity to S1. Neuroimage 127, 363-375.

Vidoni, E.D., Acerra, N.E., Dao, E., Meehan, S.K., Boyd, L.A., 2010. Role of the primary somatosensory cortex in motor learning: an rTMS study. Neurobiol. Learn. Mem. 93, 532-539.

Zangaladze, A., Epstein, C.M., Grafton, S.T., Sathian, K., 1999. Involvement of visual cortex in tactile discrimination of orientation. Nature 401, 587-590.

Zangenehpour, S., Zatorre, R.J., 2010. Crossmodal recruitment of primary visual cortex following brief exposure to bimodal audiovisual stimuli. Neuropsychologia 48 , 591-600.

Zeki, S., Watson, J.D., Lueck, C.J., Friston, K.J., Kennard, C., Frackowiak, R.S., 1991. A direct demonstration of functional specialization in human visual cortex.

J. Neurosci. 11, 641-649. 